\title{
PHOTOGRAMMETRIC ANALYSIS OF HISTORICAL IMAGE REPOSITORIES FOR VIRTUAL RECONSTRUCTION IN THE FIELD OF DIGITAL HUMANITIES
}

\author{
F. Maiwald ${ }^{\text {a }}$, T. Vietze ${ }^{\text {a }}$, D. Schneider ${ }^{\text {a }}$, F. Henze ${ }^{\text {b }}$, S. Münster ${ }^{\text {c }}$, F. Niebling ${ }^{\text {d }}$
a Institute of Photogrammetry and Remote Sensing, Technische Universität Dresden, Germany - theresa.vietze@ mailbox.tu- dresden.de, (danilo.schneider, ferdinand.maiwald)@tu-dresden.de
${ }^{\mathrm{b}}$ Informationsverarbeitung im Bauwesen/Vermessungskunde, Brandenburgische Technische Universität Cottbus-Senftenberg, Germany - frank.henze@b-tu.de
${ }^{\mathrm{c}}$ Media Center, Technische Universität Dresden, Germany - sander.muenster@tu-dresden.de
${ }^{\mathrm{d}}$ Human-Computer Interaction, Julius-Maximilians-Universität Würzburg, Germany - florian.niebling@ uni-wuerzburg.de

\section{Commission II}

KEY WORDS: Historical images, structure-from-motion, image configuration, point cloud, virtual 3D reconstruction

\begin{abstract}
:
Historical photographs contain high density of information and are of great importance as sources in humanities research. In addition to the semantic indexing of historical images based on metadata, it is also possible to reconstruct geometric information about the depicted objects or the camera position at the time of the recording by employing photogrammetric methods. The approach presented here is intended to investigate (semi-) automated photogrammetric reconstruction methods for heterogeneous collections of historical (city) photographs and photographic documentation for the use in the humanities, urban research and history sciences. From a photogrammetric point of view, these images are mostly digitized photographs. For a photogrammetric evaluation, therefore, the characteristics of scanned analog images with mostly unknown camera geometry, missing or minimal object information and low radiometric and geometric resolution have to be considered. In addition, these photographs have not been created specifically for documentation purposes and so the focus of these images is often not on the object to be evaluated. The image repositories must therefore be subjected to a preprocessing analysis of their photogrammetric usability. Investigations are carried out on the basis of a repository containing historical images of the Kronentor ('crown gate') of the Dresden Zwinger. The initial step was to assess the quality and condition of available images determining their appropriateness for generating three-dimensional point clouds from historical photos using a structure-from-motion evaluation (SfM). Then, the generated point clouds were assessed by comparing them with current measurement data of the same object.
\end{abstract}

\section{INTRODUCTION}

In recent decades there was much research on photogrammetric reconstruction from historical and user generated content. With regards to a reconstruction from heterogeneous photographs, a focus in previous research was specially to involve large amounts of photographs from web based repositories (Ioannides et al., 2013), (Kersten et al., 2012). For example, Snavely et al. (2007) have examined the reconstruction of the Great Wall of China from various internet photo collections. Moreover, historical photography has been used to reconstruct lost heritage in various projects, sometimes with broad recognition, for instance the reconstruction of the Buddha statues in Bamiyan Afghanistan which were destroyed in the early 2000s (Grün et al., 2004). Most of these projects are dealing with photographs not more than a few decades old, only a small number of projects rely heavily on photographs which are more than 50 years old (Gouveia et al., 2015), (Henze et al., 2009). One example is the reconstruction of the Paluxy River Tracks from 1940s photographs by Roland T. Bird from (Falkingham et al., 2014).

Even if basic workflows are independent of the age of the photograph or creation process - digital or non-digital - by which it was created, older photographs, are more challenging to work with due to archival quality, issues of digitization, technical equipment and film media. Another challenge relates to the context and purpose of photographs in digital image repositories. Since there is much research on several purposes of photographic production- e.g. for documentation of architecture, press, personal memories - in the field of visual studies (Remondino et al., 2012), these aspects can affect photogrammetric reconstruction of architecture with regards for instance to completeness of photographic documentation or 'occlusions' in images caused for example by persons or cars.

This contribution presents an approach to reconstructing an object which was commonly photographed at different times. Photographs used in this paper come from the photo library of the Saxon State and University Library Dresden (SLUB). The historical images used show parts of the Dresden Zwinger, especially the Kronentor. Firstly the hits in the photo library were filtered and sorted manually by means of sources (maps, plans, photography) and for general image quality. Furthermore the characteristics of scanned analogue images with unknown camera geometry, differing radiometric resolution or even destroyed image sources had to be considered. In the next step the photogrammetric quality of the photos for the threedimensional reconstruction with structure-from-motion (SfM) was evaluated. The rear and front sides of the Kronentor were separated in this investigation because of a lack of tie points between the two sides. In the future these two parts will be combined.

In a second approach the historical photos were processed with additional current images. This made it possible to use more 
historical images for the SfM calculation and thus to generate a denser point cloud. Finally the quality of the generated historical point cloud was compared to a present SfM point cloud and a point cloud created by terrestrial laser scanning.

The investigated object is part of the Zwinger - one of the most visited sights of the city of Dresden. The Kronentor forms the western entrance of the palace complex. Both sides are affiliated with a long gallery, which are enclosed in front by a moat. Over this moat leads a bridge to the Kronentor. It was built in 1714 as a gate with an additional tower in the baroque style. Two pairs of columns form the main component of the passage. The gate is two-storied, and the upper floor can only be reached through the roof corridor of the wings. A parapet wall encloses the hall in the upper floor, which is open on four sides. The Kronentor is completed by a bulbous dome with a golden crown.

In 1945 large sections of the Zwinger, including the Kronentor, were heavily damaged by bombs. It was reconstructed after the war, and later portico with gold fittings was added to the dome. Between 2012 and 2016 the Kronentor was refurbished completely. With the help of historical images and photogrammetric methods, the original building can be reconstructed. In doing so, it is possible to reveal structural changes in comparison to current models.

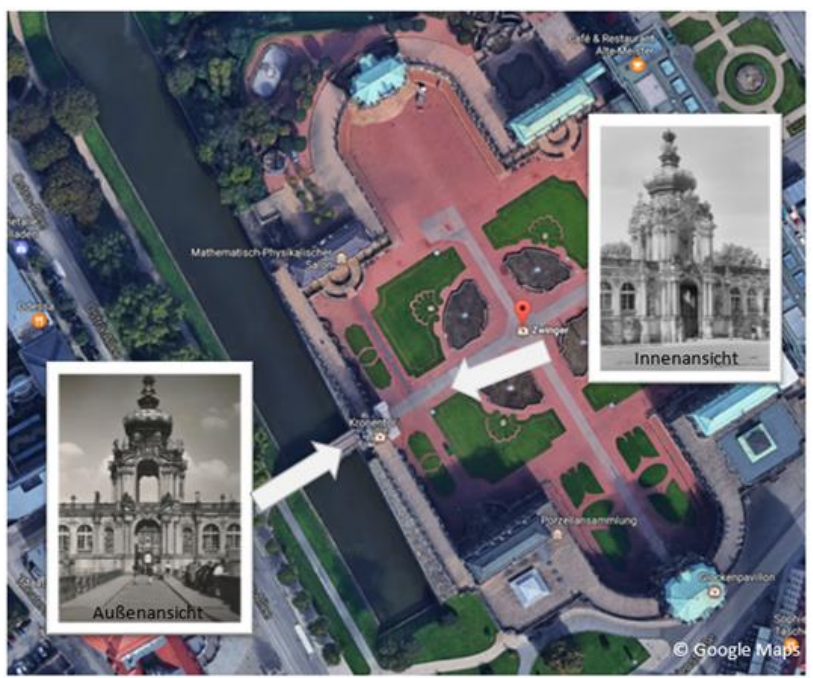

Figure 1. Overview of the Zwinger with historical images of the Kronentor (Google maps)

\section{DATA SOURCES}

In order to find suitable images of the Dresden Zwinger, more than 800 historical photos from different periods were filtered from the photo library of the Saxon State and University Library Dresden (SLUB) by a keyword search. The data set could be reduced to 499 images by more detailed search criteria. At first sight, this is a very large data base, but this was limited to 44 photographs by applying further selection criteria. These criteria can be divided into two groups. Firstly, all recordings which do not appear to show the Kronentor or are not suitable for a later 3D reconstruction using structure-from-motion (SfM) were removed. These included drawings, postcards and sketches. Night shots and recordings, which depict the Kronentor scaffolded were also rejected, as this would affect the image matching results. Recordings at a greater distance from the object do not provide the necessary level of detail for a 3D reconstruction. An image segment should also show the Kronentor at full size. Considering these factors the data set could be reduced from 499 to 89 images, and then reduced further by assessing the quality of the photos. Images with a low scan resolution are often not suitable for SfM, since certain details are not represented adequately. As a result, too few feature points were found, which means that the image orientation may be computed inaccurately, or not at all. In the subsequent dense matching process, many wrong point assignments were found, which led to outliers within the final point cloud. Radiation or blurring will also distort the result. A few pictures had to be deselected because they were warped or contained kinks. Finally, a data set with 44 images was created (compare examples in Fig. 2). It was assumed that this data set was in principle suitable for the generation of point clouds using SfM.

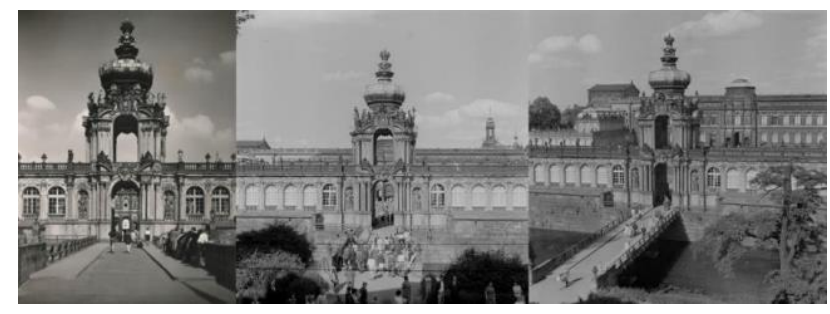

Figure 2. Examples for historical images of the Kronentor used for SfM

Taking a closer look at the selected images, however, it was found that many recordings are taken from the same imaging direction, whereas pictures from other perspectives are not available. The historical photographs are taken mainly by private persons or newspaper photographers, who did not photograph the Kronentor according to photogrammetric criteria. This is a problem for SfM, since the overlapping areas between photos from two different imaging positions are not sufficient to be able to link them together. In addition, the baseline between the images is too large to reliably determine homologous image points. Therefore, images of interjacent positions are required, but they either do not exist or their resolution is too low. The local conditions also restricted the photographer's choice of shooting location. The recordings can be subdivided into two groups: those made from outside or inside the Zwinger (Fig. 1). A point cloud was generated for each of these groups. The two point clouds could be transferred into a common coordinate system at a later stage.

\section{DATA PROCESSING AND RESULTS}

Applying Agisoft PhotoScan it was evaluated whether point clouds can be created from historical images with SfM (Lowe, 2004). Through previous research and experience, this program promised the best results (Remondino et al., 2012), (Santagati et al., 2013). Other software, such as VisualSfM, was also tested. However, the comparison of the programs should not be the focus here.

The investigation of the point clouds created from the previously selected historical recordings is described in this section. Some of the point clouds represent the side of the Kronentor, which is outside the Dresden Zwinger. Another point cloud represents the object area visible from the courtyard (compare Fig. 1). The calibration of the cameras was determined by the self-calibration within PhotoScan. The 
generated point clouds are still arbitrarily scaled and are not registered in a global coordinate system.

\subsection{Generation of a point cloud exclusively using historical images}

To create the point cloud which represents the Kronentor from outside, at first 25 images with a subjective high quality were imported into PhotoScan. However, in the workflow it was noted that some images did not have enough overlapping areas with others. Therefore, only 11 images could be automatically orientated and then used to generate the point cloud (Fig. 3).

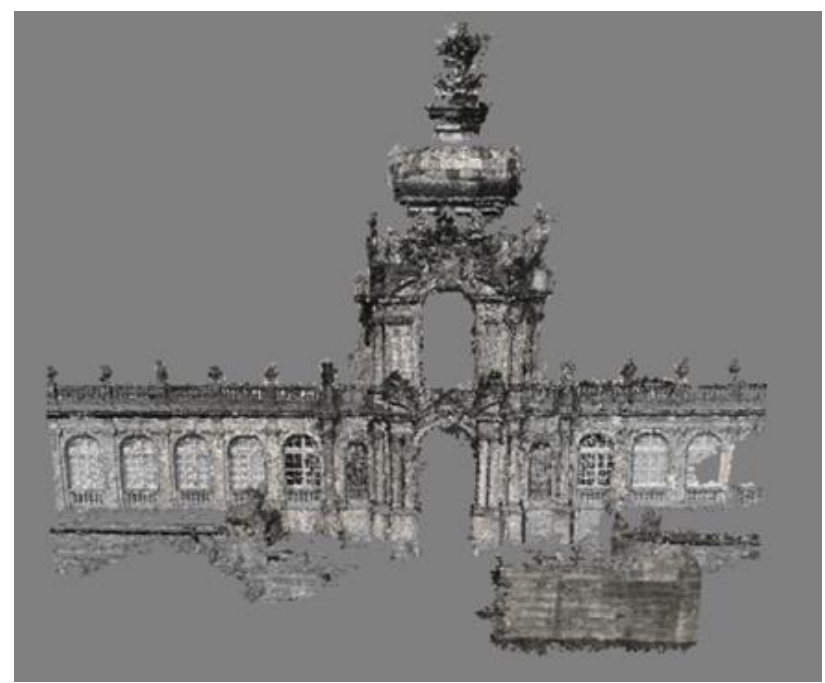

Figure 3. Point cloud generated from historical images (exterior view of the Zwinger)

First of all, observing the point cloud, the Kronentor can be recognized. Small details, such as sandstone figures on the wings are only visible schematically (compare Fig. 4). This is because the given resolution of the images does not comprise these fine details. During the reconstruction of the Dresden Zwinger and the Kronentor after the damage of 1945, small structural details were altered or replaced. The exact years when images were recorded are not known. It can therefore be assumed that images from different periods of the building's history were integrated into the image block. The structural changes led to reconstruction errors.

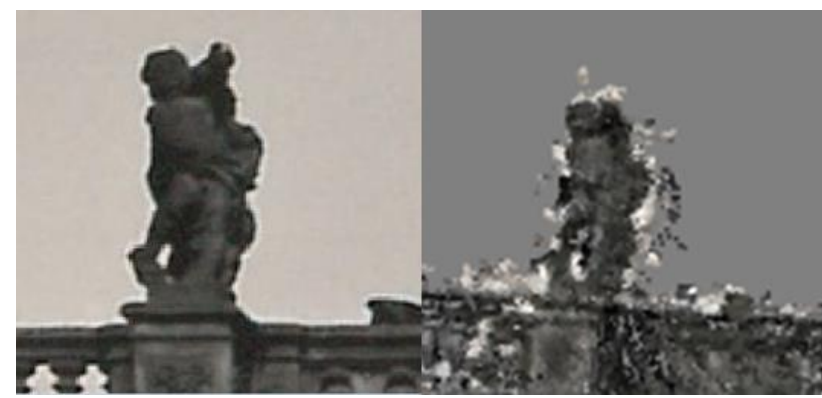

Figure 4. Comparison of a segment originated from a historical image (left) and a point cloud (right)

It should be noted that the selected photographs were mostly taken from a central position (Fig. 5). Therefore, on the one hand, the model is incomplete in some places due to occlusions. On the other hand, the very short baselines between the camera positions lead to small intersection angles and thus to low accuracy of the computed object points. In order to achieve better results, a better imaging configuration is crucial. For this purpose, the images taken from other viewing directions, which could not be oriented initially, must be included as well.

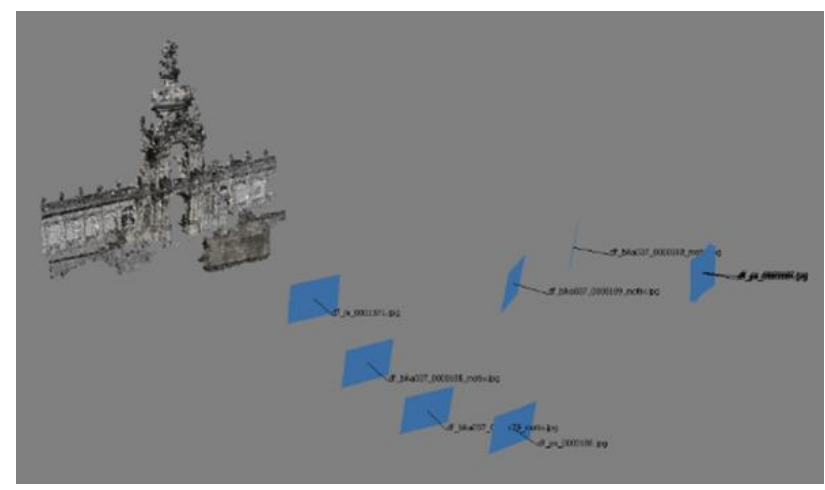

Figure 5. Historical point cloud with camera positions of the images used

The second point cloud is based on photographs taken from within the Dresden Zwinger. However, it has to be noted that only photographs taken from the left-hand direction of view were suitable. Recordings from other perspectives, which could strengthen the imaging geometry, were not included due to their low resolution. Some of the images used have also been taken from a longer distance (Fig. 7), so the given resolution on the object is not adequate to recognize details (Fig. 6). In order to achieve a satisfactory result, analogue photographs with a significantly higher resolution need to be digitalized. This assumes that the analogue originals offer a higher resolution potential than the existing digitization. However, it can be assumed that a high-resolution scan is available in many cases, since until this point the preview image of the SLUB photo library was often used.

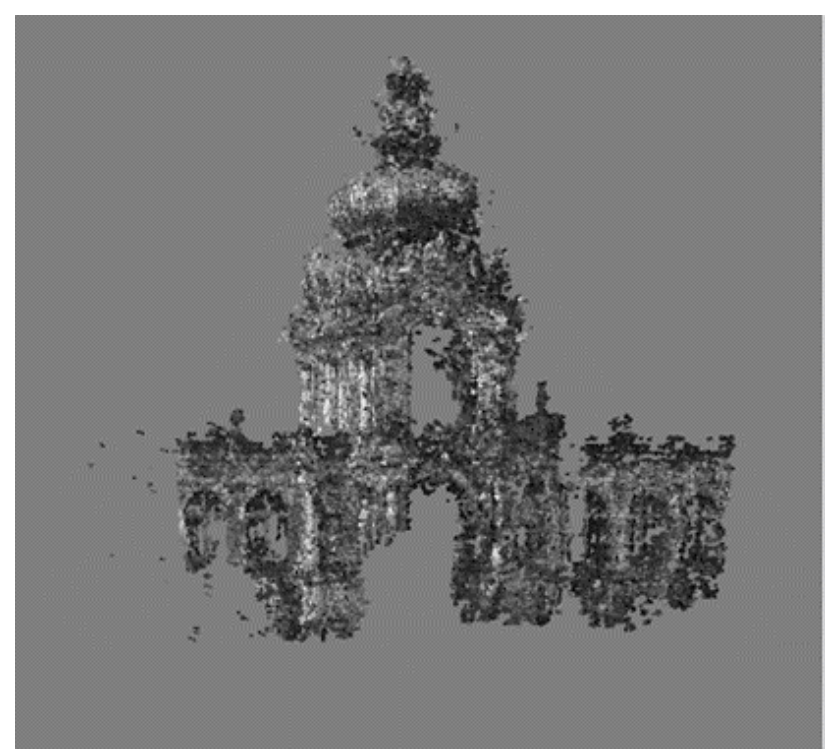

Figure 6. Historical point cloud (interior view of the Zwinger) 


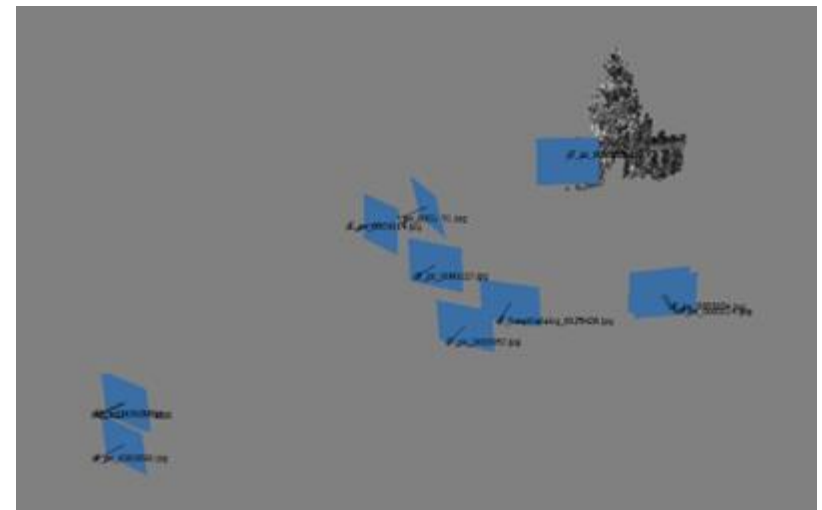

Figure 7. Camera positions for the interior point of view

\subsection{Generation of a point cloud integrating historical and current images}

The following investigations relate only to the side of the Kronentor that is visible from the outside of the Dresden Zwinger (see Section 3.1). For the orientation of further historical images with more suitable imaging directions, current images are used as 'filling material', since they provide smaller baselines between the images (Fig. 8).

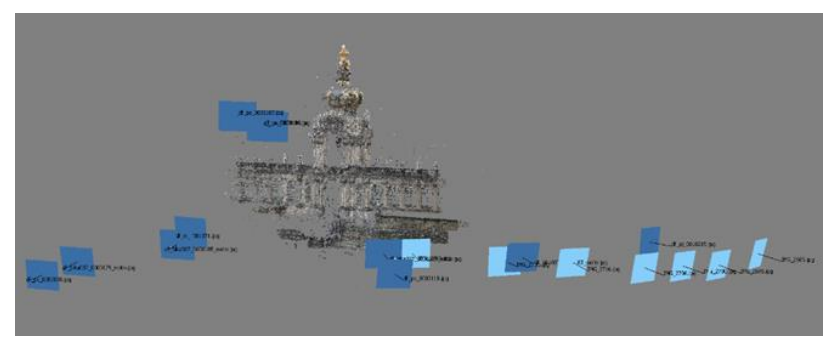

Figure 8. Camera positions of the historical (dark blue) and the current (light blue) images

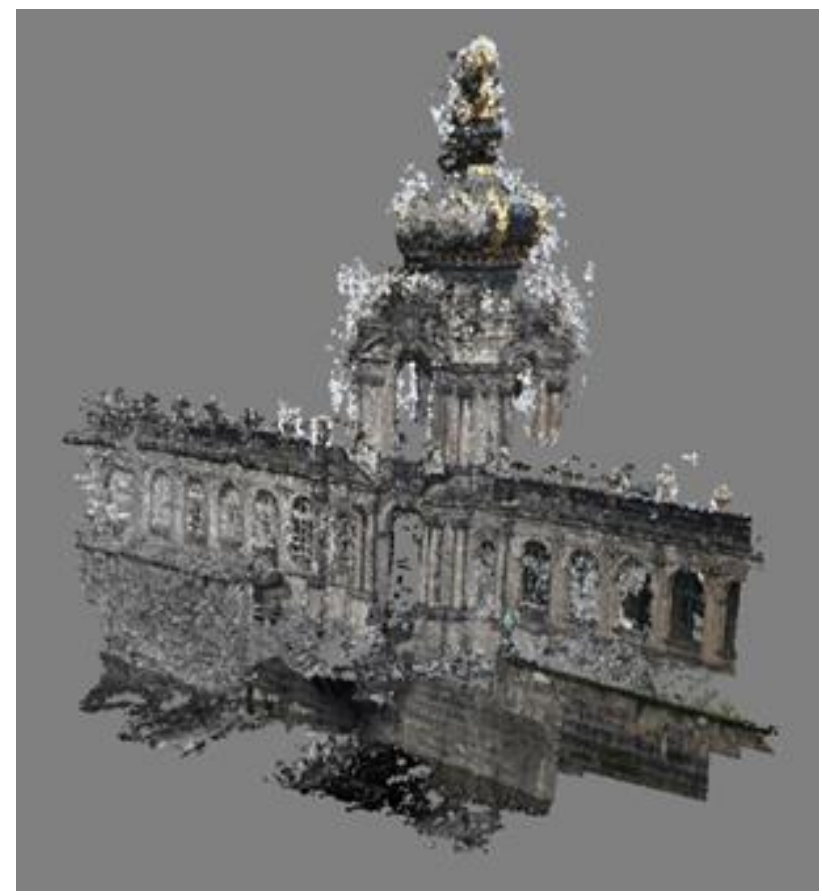

Figure 9. Point cloud generated on the basis of both, historical as well as current images
Thus, historical images, which are located at the edge, can be orientated and subsequently included in the processing of the point cloud.

Figure 9 shows that the level of detail and the number of points in the point cloud have increased significantly (from approx. 1.3 to 3 million points in the dense cloud). However, the 'historical points' are superimposed with 'current points'. This can be clearly seen by the structural changes to the crown. The historical recordings do not show the gold fittings on the roof. Thus, the current images distort the historical point cloud. Therefore, these points have to be removed, which is described in more detail in the following section.

\subsection{Generation of a point cloud from historical images with current images supporting the image orientation of historical images}

In the following, a point cloud is to be generated in which the current images were only included to support the determination of the orientation of the historical images. One option for doing this is to import the previously computed orientation of an image (see Section 3.2) into a new PhotoScan project. This means that only historical recordings are used throughout the workflow. Another option is to use the project, in which historical and current images are combined and specify that the current photos should only be taken into account for the image orientation (and the generation of the sparse point cloud) but not for the following step, in which the dense cloud is processed. In both cases, all points in the resulting point cloud originate exclusively from the historical photos. Using this procedure, two other historical photos could be integrated into the image block. This made it possible to fill some gaps in the first point cloud (Section 3.1) and the level of detail could be improved in some object areas (Fig. 3, Fig. 10). The number of points in the dense point cloud could also be increased from approximately 1.3 to 1.7 million.

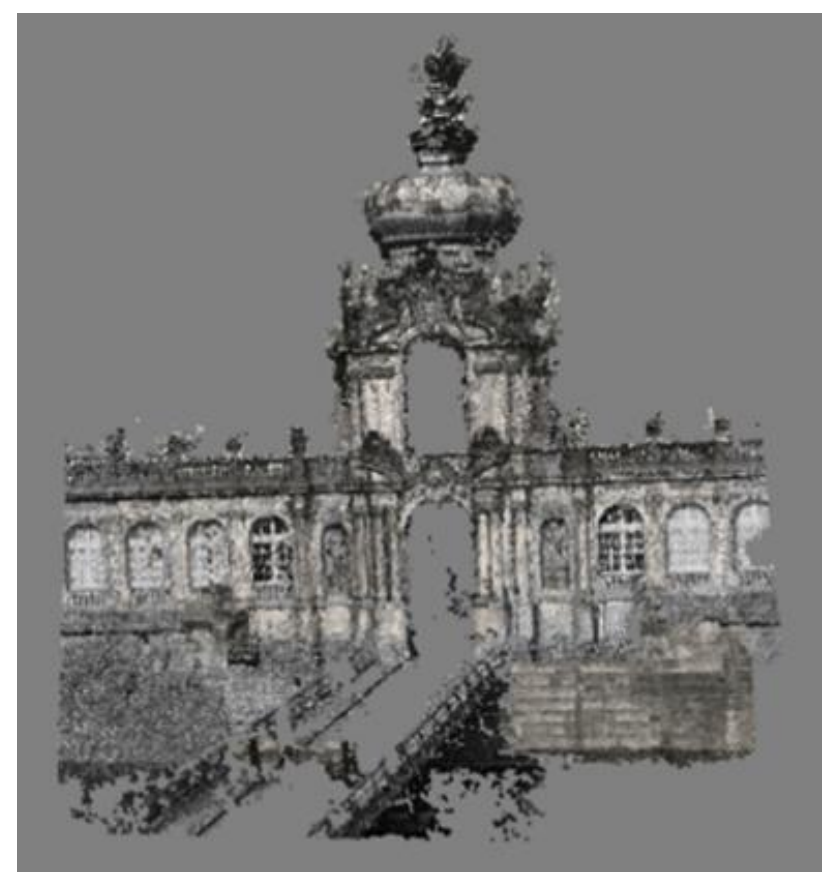

Figure 10. Point cloud based on 13 historical images 


\subsection{Comparison of the point cloud with a point cloud from recent images and terrestrial laser scanner data}

The point cloud generated from historical images (Fig. 10) is compared with the point cloud, which was created from 40 exclusively current images (Fig. 11). In both cases, the same settings have been used in PhotoScan. A visual comparison clearly shows that the current point cloud is less noisy and more details are reproduced. A third point cloud (Fig. 12) was generated with a terrestrial laser scanner Riegl LMS-Z420i from a total of 6 points around the Kronentor. The laser scanning point cloud, which is not yet colorized, shows the highest level of detail and the lowest noise.

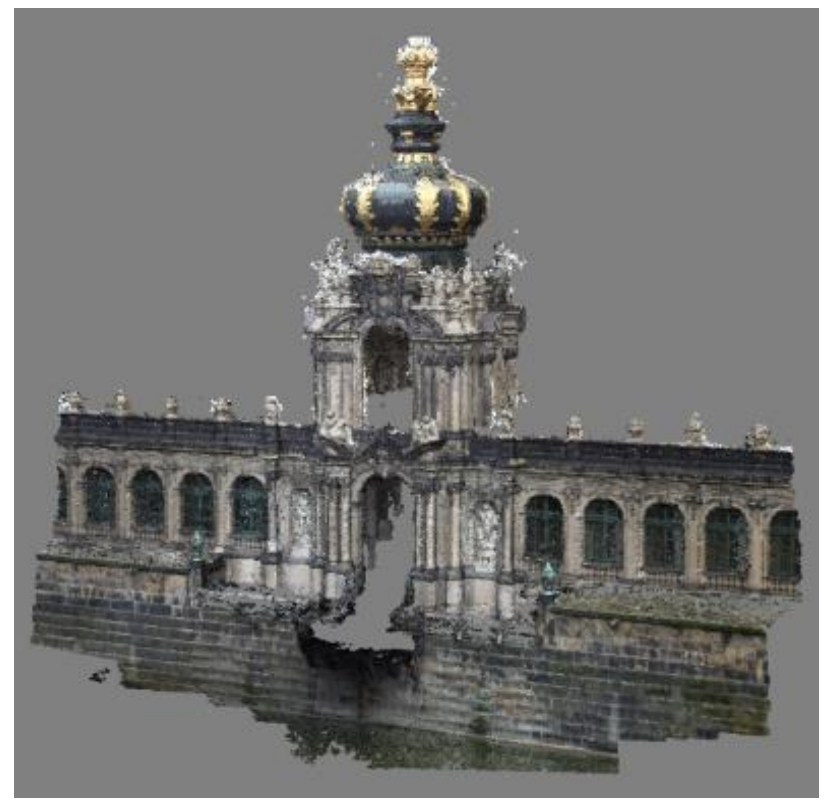

Figure 11. Point cloud based on recent images

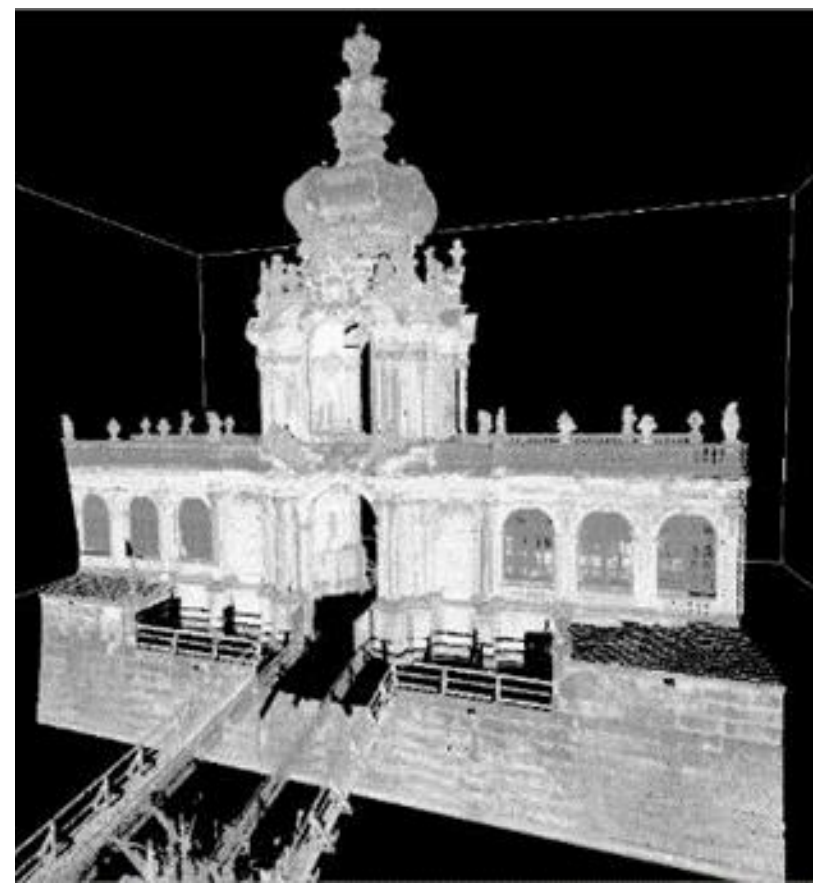

Figure 12. Point cloud based on terrestrial laser scanner data

\section{CONCLUSION AND FUTURE RESEARCH}

It is possible to generate three-dimensional point clouds from historical, digitalized photographs using structure-from-motion (SfM). Furthermore triangulated and textured 3D models can be generated on the basis of these point clouds. This paper has shown the requirements to be met by historical photographs and how the SfM workflow can be influenced in order to create point clouds or models with a high visual quality. Factors which have to be taken into account include imaging configuration, camera parameters, camera resolution and image quality.

The paper discusses additional alternative methods for generating 3D models, such as the integrated processing of historical and current images. This approach can only be used if the architecture of the observed building has not significantly changed over time. And it has to be possible to reach the object with photogrammetric methods currently. In case of a small number of images or too long baselines between the camera positions, partial point clouds of the object were generated. In a following processing step partial models can be potentially merged.

Further investigations could include digitalized photography with a higher scanning resolution. This would probably improve the quality of point clouds generated by these images significantly. In future research, objective quality metrics, e.g. for accuracy and completeness will be defined which can be used to compare and to analyse the quality of different point clouds.

If no better-quality images exist an approach could be evaluated based on interactive modelling of object geometries. This approach might be more suitable due to a potential higher visual quality of the results it the targeted area of application is history teaching or history research. However, it would require more user interaction and thus, the results would depend stronger on the modeler's knowledge and assumptions about the object in this case. Orientation of individual historical images just for object texturing of virtual 3D models could be another option.

\section{ACKNOWLEDGEMENTS}

This research is part of ongoing investigations of the junior research group HistStadt4D, comprising 14 people - including 4 post-doctoral and $4 \mathrm{PhD}$ researchers - which are working until July 2020 on how digital image archives and media repositories can be used for urban historical research and on how to communicate the results of this research.

\section{REFERENCES}

Falkingham, P., L., Bates, K., T. and Farlow, J., O., 2014. Historical Photogrammetry: Bird's Paluxy River Dinosaur Chase Sequence Digitally Reconstructed as It Was prior to Excavation 70 Years Ago. PLoS ONE, 9(4): e93247.

Gouveia, J., Branco, F., Rodrigues, A. and Correia, N., 2015. Travelling through space and time in Lisbon's religious buildings. 2015 Digital Heritage, Granada, pp. 407-408.

Grün, A., Remondino, F. and Zhang, L. 2004. Photogrammetric Reconstruction of the Great Buddha of Bamiyan, Afghanistan. The Photogrammetric Record, 19, pp. 177-199. 
Henze, F., Lehmann, H. and Bruschke, B., 2009. Nutzung historischer Pläne und Bilder für dieStadtforschungen in Baalbek / Libanon. In: Photogrammetrie - Fernerkundung Geoinformation (PFG), 3/2009, pp. 221-234.

Ioannides, M., Hadjiprocopi, A., Doulamis, N., Doulamis, A., Protopapadakis, E., Makantasis, K., Santos, P., Fellner, D., Stork, A., Balet, O., Julien, M., Weinlinger, G., Johnson, P. S., Klein, M. and Fritsch, D., 2013. Online 4D Reconstruction Using Images Available Under Open Access. ISPRS Ann. Photogramm. Remote Sens. Spatial Inf. Sci., II-5/W1, pp. 169174.

Kersten, T., Lindstaedt, M., Michelke, K. and Zobel, K., 2012. Automatische 3D-Objektrekonstruktion aus unstrukturierten, digitalen Bilddaten für Anwendungen in Architektur, Denkmalpflege und Archäologie. Publikationen der deutschen Gesellschaft für Photogrammetrie, Fernerkundung und Geoinformation $e$. V., Band 21, 32. WissenschaftlichTechnische Jahrestagung der DGPF, pp. 137-148.

Lowe, D., G., 2004: Distinctive Image Features from ScaleInvariant Keypoints. International Journal of Computer Vision 60 (2), pp. 91-110.

Remondino, F., Pizzo, S., D., Kersten T., P. and Troisi, S., 2012. Low-Cost and Open-Source Solutions for Automated Image Orientation - A Critical Overview. In: Ioannides M (ed) EUROMED 2012, pp 40-54.

Santagati, C., Inzerillo, L. and Di Paola, F., 2013: Image-based modeling techniques for architectural heritage 3D digitalization: Limits and potentialities. International Archives of the Photogrammetry, Remote Sensing and Spatial Information Sciences 40 (5/W2), pp. 555-560.

Snavely, N., Seitz, S., M. and Szeliski, R., 2007. Modeling the World from Internet Photo Collections. International Journal of Computer Vision, 80 (2), pp.189-210. 